\title{
The Abel Integral Equations in Distribution
}

\author{
Chenkuan Li ${ }^{1 *}$, Changpin Li ${ }^{2}$, Bailey Kacsmar ${ }^{1}$, Roque Lacroix ${ }^{1}$ and Kyle Tilbury ${ }^{1}$ \\ ${ }^{1}$ Department of Mathematics and Computer Science, Brandon University, Brandon, Manitoba, Canada R7A 6A9 \\ ${ }^{2}$ Department of Mathematics, Shanghai University, Shanghai 200444, China \\ Email: lic@brandonu.ca
}

\begin{abstract}
This paper is to study the following generalized Abel's integral equation

$$
g(x)=\frac{1}{\Gamma(1-\alpha)} \int_{0}^{x} \frac{f(\zeta)}{(x-\zeta)^{\alpha}} d \zeta, \quad \text { where } \alpha \in R
$$

and its variant in the distributional (Schwartz) sense based on fractional calculus of distributions. We obtain a number of interesting and new results which are not achievable in the classical sense.

Keywords: Distribution, convolution, delta function, Abel's equation, Gamma function, Caputo derivative and Riemann-Liouville derivative.
\end{abstract}

\section{Introduction}

An integral equation is an equation in which an unknown function appears under an integral sign. There is a close connection between differential and integral equations in general, and some problems can be formulated either way. Integral equations play an important role in both pure and applied mathematics and they also have numerous applications in physics, chemistry, biology, electronics and mechanics ([1], [2] and [3]). In 1823, N. Abel studied a physical problem about the relationship between kinetic and potential energies for falling objects in [4] and obtained an integral stated in the form

$$
g(x)=\int_{a}^{x} \frac{f(\zeta)}{\sqrt{x-\zeta}} d \zeta, \quad a>0
$$

where $g$ is given and $f$ is unknown. He also worked on a more general integral equation given as

$$
g(x)=\frac{1}{\Gamma(1-\alpha)} \int_{0}^{x} \frac{f(\zeta)}{(x-\zeta)^{\alpha}} d \zeta, \quad 0<\alpha<1, a \leq x \leq b,
$$

which is called the classical Abel's integral equation or integral equation of Abel's type.

There are many approaches to solving the classical Abel's integral equations, including their variants, with applications. In 1930, Tamarkin [5] discussed integrable solutions of Abel's integral equations under certain conditions by several integral operators. Sumnar [6] studied Abel's integral equations from the point of the convolution transform. Minerbo and Levy [7] investigated numerical solutions of Abel's integral equations using orthogonal polynomials. In 1985, Hatcher [8] worked on a nonlinear Hilbert problem of power type, solved in closed form by representing a sectionally holomorphic function by means of an integral with power kernel, and he transformed the problem to one of solving a generalized Abel's integral equation. Singh et al. [9] obtained stable numerical solutions of Abel's integral equations using almost Bernstein operational matrix. Recently, Li and Zhao [10] used the inverse of Mikusinski's operator of fractional order based on Mikusinski's convolution to construct the solutions of integral equations of Abel's type. Jahanshahi et al. [11] solved Abel's integral equations numerically based on approximations of fractional integrals and Caputo derivatives. Saleh et al. [12] investigated the numerical solutions of Abel's integral equations by Chebyshev polynomials. There are many other research papers dealing with Abel's equations and more beginning to appear.

The structure of the current work is grouped as follows. In section 2, we briefly introduce the necessary concepts and definitions of fractional calculus of distributions in $\mathcal{D}^{\prime}\left(R^{+}\right)$, and further we show that the Riemann-Liouville derivative and Caputo derivative of distributions are identical by distributional 
convolutions. In section 3, we solve the generalized Abel's integral equation for all $\alpha \in R$ and its variant in the distributional space $\mathcal{D}^{\prime}\left(R^{+}\right)$by a new technique of computing fractional derivatives of distributions based on derivatives of integer-order. We derive some novel results for all $\alpha \in R$ which cannot be not realized in the classical sense.

\section{Distributions in $\mathcal{D}^{\prime}\left(R^{+}\right)$}

In order to study fractional derivatives of certain types of distributions and proceed smoothly, we introduce the following basic concepts in detail, which will be used in the subsequent section. Let $\mathcal{D}(R)$ be the Schwartz space [13] of infinitely differentiable functions with compact support in $R$, and $\mathcal{D}^{\prime}(R)$ be the space of distributions defined on $\mathcal{D}(R)$. Further, we shall define a sequence $\phi_{1}(x), \phi_{2}(x), \cdots, \phi_{n}(x), \cdots$ which converges to zero in $\mathcal{D}(R)$ if all these functions vanish outside a certain fixed bounded interval, and converge uniformly to zero (in the usual sense) together with their derivatives of any order. The functional $\delta(x)$ is defined as

$$
(\delta, \phi)=\phi(0)
$$

where $\phi \in \mathcal{D}(R)$. Clearly, $\delta$ is a linear and continuous functional on $\mathcal{D}(R)$, and hence $\delta \in \mathcal{D}^{\prime}(R)$. Let $\mathcal{D}\left(R^{+}\right)$be the subspace of $\mathcal{D}^{\prime}(R)$ with support contained in $R^{+}$.

Define

$$
\theta(x)=\left\{\begin{array}{l}
1 \text { if } x>0 \\
0 \text { if } x<0
\end{array}\right.
$$

Then

$$
(\theta(x), \phi(x))=\int_{0}^{\infty} \phi(x) d x \quad \text { for } \quad \phi \in \mathcal{D}(R),
$$

which implies $\theta(x) \in \mathcal{D}^{\prime}(R)$.

Let $f \in \mathcal{D}^{\prime}(R)$. The distributional derivative of $f$, denoted by $f^{\prime}$ or $d f / d x$, is defined as

$$
\left(f^{\prime}, \phi\right)=-\left(f, \phi^{\prime}\right)
$$

for $\phi \in \mathcal{D}(R)$.

Clearly, $f^{\prime} \in \mathcal{D}^{\prime}(R)$ and every distribution has a derivative. As an example, we are going to show that $\theta^{\prime}(x)=\delta(x)$, although $\theta(x)$ is not even defined at $x=0$. Indeed,

$$
\left(\theta^{\prime}(x), \phi(x)\right)=-\left(\theta(x), \phi^{\prime}(x)\right)=-\int_{0}^{\infty} \phi^{\prime}(x) d x=\phi(0)=(\delta(x), \phi(x))
$$

which claims

$$
\theta^{\prime}(x)=\delta(x)
$$

It can be shown that the ordinary rules of differentiation apply also to distributions. For instance, the derivative of a sum is the sum of the derivatives, and a constant can be commuted with the derivative operator.

It follows from [13] and [14] that $\Phi_{\lambda}=\frac{x_{+}^{\lambda-1}}{\Gamma(\lambda)} \in \mathcal{D}^{\prime}\left(R^{+}\right)$is an entire function of $\lambda$ on the complex plane, and

$$
\left.\frac{x_{+}^{\lambda-1}}{\Gamma(\lambda)}\right|_{\lambda=-n}=\delta^{(n)}(x), \quad \text { for } \quad n=0,1,2, \cdots
$$

For the functional $\Phi_{\lambda}=\frac{x_{+}^{\lambda-1}}{\Gamma(\lambda)}$, the derivative formula is simpler than that for $x_{+}^{\lambda}$. In fact,

$$
\frac{d}{d x} \Phi_{\lambda}=\frac{d}{d x} \frac{x_{+}^{\lambda-1}}{\Gamma(\lambda)}=\frac{(\lambda-1) x_{+}^{\lambda-2}}{\Gamma(\lambda)}=\frac{x_{+}^{\lambda-2}}{\Gamma(\lambda-1)}=\Phi_{\lambda-1} .
$$

The convolution of certain pairs of distributions is usually defined as follows, see Gel'fand and Shilov [13] for example.

Definition 2.1 Let $f$ and $g$ be distributions in $\mathcal{D}^{\prime}(R)$ satisfying either of the following conditions: 
(a) either $f$ or $g$ has bounded support (set of all essential points), or

(b) the supports of $f$ and $g$ are bounded on the same side.

Then the convolution $f * g$ is defined by the equation

$$
((f * g)(x), \phi(x))=(g(x),(f(y), \phi(x+y)))
$$

for $\phi \in \mathcal{D}(R)$.

The classical definition of the convolution is as follows:

Definition 2.2 If $f$ and $g$ are locally integral functions, then the convolution $f * g$ is defined by

$$
(f * g)(x)=\int_{-\infty}^{\infty} f(t) g(x-t) d t=\int_{-\infty}^{\infty} f(x-t) g(t) d t
$$

for all $x$ for which the integrals exist.

Note that if $f$ and $g$ are locally integrable functions satisfying either of the conditions in (a) or (b) in Definition 2.1, then Definition 2.1 is in agreement with Definition 2.2. It also follows that if the convolution $f * g$ exists by Definition 2.1 or 2.2 , then the following equations hold:

$$
\begin{aligned}
& f * g=g * f \\
& (f * g)^{\prime}=f * g^{\prime}=f^{\prime} * g
\end{aligned}
$$

where all the derivatives above are in the distributional sense.

Let $\lambda$ and $\mu$ be arbitrary complex numbers. Then it is easy to show

$$
\Phi_{\lambda} * \Phi_{\mu}=\Phi_{\lambda+\mu}
$$

by equation (4), without any help of analytic continuation mentioned in all current books.

Let $\lambda$ be an arbitrary complex number and $g(x)$ be the distribution concentrated on $x \geq 0$. We define the primitive of order $\lambda$ of $g$ as convolution in the distributional sense

$$
g_{\lambda}(x)=g(x) * \frac{x_{+}^{\lambda-1}}{\Gamma(\lambda)}=g(x) * \Phi_{\lambda} .
$$

Note that the convolution on the right-hand side is well defined since supports of $g$ and $\Phi_{\lambda}$ are bounded on the same side.

Thus equation (8) with various $\lambda$ will not only give the fractional derivatives, but also the fractional integrals of $g(x) \in \mathcal{D}^{\prime}\left(R^{+}\right)$when $\lambda \notin Z$, and it reduces to integer-order derivatives or integrals when $\lambda \in Z$. We shall define the convolution

$$
g_{-\lambda}=g(x) * \Phi_{-\lambda}
$$

as the fractional derivative of the distribution $g(x)$ with order $\lambda$, writing it as

$$
g_{-\lambda}=\frac{d^{\lambda}}{d x^{\lambda}} g
$$

for $\operatorname{Re} \lambda \geq 0$. Similarly, $\frac{d^{\lambda}}{d x^{\lambda}} g$ is interpreted as the fractional integral if $\operatorname{Re} \lambda<0$.

For a given function, its classical Riemann-Liouville derivative or Caputo derivative ([15], [16] and [10]) may not exist in general ([17], [18] and [19]). Even if they do, the Riemann-Liouville derivative and the Caputo derivative are not necessarily the same. However, if $g(x)$ is a distribution in $\mathcal{D}^{\prime}\left(R^{+}\right)$, then the case is different. Let $m-1<\operatorname{Re} \lambda<m \in Z^{+}$. From equation (6), we derive that

$$
\begin{aligned}
g_{-\lambda}(x) & =g(x) * \frac{x_{+}^{-\lambda-1}}{\Gamma(-\lambda)}=g(x) * \frac{d^{m}}{d x^{m}} \frac{x_{+}^{m-\lambda-1}}{\Gamma(m-\lambda)} \\
& =\frac{d^{m}}{d x^{m}}\left(g(x) * \frac{x_{+}^{m-\lambda-1}}{\Gamma(m-\lambda)}\right)=\frac{x_{+}^{m-\lambda-1}}{\Gamma(m-\lambda)} * g^{(m)}(x),
\end{aligned}
$$

which indicates there is no difference between the Riemann-Liouville derivative and the Caputo derivative of the distribution $g(x)$ (both exist clearly). Based on this fact, we only call the fractional derivative of distribution for brevity. 


\section{The Abel's Integral Equations}

Theorem 3.1 Let $g(x)$ be given in $\mathcal{D}^{\prime}\left(R^{+}\right)$and $f(x)$ be unknown in $\mathcal{D}^{\prime}\left(R^{+}\right)$. Then the generalized Abel's integral equation given by

$$
g(x)=\frac{1}{\Gamma(1-\alpha)} \int_{0}^{x} \frac{f(\zeta)}{(x-\zeta)^{\alpha}} d \zeta
$$

has the solution

$$
f(x)=g(x) * \Phi_{\alpha-1}
$$

where $\alpha$ is any real number. In particular, if $-m<\alpha<-m+1$ for $m \in Z^{+}$then

$$
f(x)=g^{(m+1)}(x) * \frac{x_{+}^{\alpha+m-1}}{\Gamma(\alpha+m)} .
$$

Proof. In the classical theory, we assume that $\alpha<1$ to ensure that the integral converges. We, however, need not make this assumption here, since the right-hand side can be understood as the following convolution in the distributional sense

$$
g(x)=f(x) * \Phi_{1-\alpha} .
$$

This implies that

$$
g(x) * \Phi_{\alpha-1}=f(x) *\left(\Phi_{1-\alpha} * \Phi_{\alpha-1}\right)=f(x) * \delta(x)=f(x)
$$

by equations (3) and (7). Assuming $-m<\alpha<-m+1$ for $m \in Z^{+}$, we have

$$
\Phi_{\alpha-1}=\frac{d^{m+1}}{d x^{m+1}} \frac{x_{+}^{\alpha+m-1}}{\Gamma(\alpha+m)}
$$

where $\frac{x_{+}^{\alpha+m-1}}{\Gamma(\alpha+m)}$ is an ordinary function. Therefore,

$$
f(x)=\frac{d^{m+1}}{d x^{m+1}}\left(g(x) * \frac{x_{+}^{\alpha+m-1}}{\Gamma(\alpha+m)}\right)=g^{(m+1)}(x) * \frac{x_{+}^{\alpha+m-1}}{\Gamma(\alpha+m)}
$$

in the distributional sense. In particular, for $m=0$ (i.e., $0<\alpha<1$ ) we have for a differentiable $g(x)$ that

$$
f(x)=g^{\prime}(x) * \Phi_{\alpha}=\frac{1}{\Gamma(\alpha)} \int_{0}^{x} \frac{g^{\prime}(\zeta)}{(x-\zeta)^{1-\alpha}} d \zeta
$$

which is the classical Abel's integral equation. This completes the proof of theorem.

Theorem 3.2 The generalized Abel's integral equation

$$
g(x)=\frac{1}{\Gamma(1-\alpha)} \int_{0}^{x} \frac{f(\zeta)}{(x-\zeta)^{\alpha}} d \zeta, \quad \alpha \in R
$$

where

$$
g(x)= \begin{cases}e^{x} & \text { if } x>0 \\ 0 & \text { if } x<0\end{cases}
$$

has the solution

$$
f(x)= \begin{cases}g(x) & \text { if } \alpha=1, \\ \delta^{(-\alpha)}(x)+\delta^{(-\alpha+1)}(x)+\cdots+\delta(x)+g(x) & \text { if } \alpha=0,-1,-2, \cdots, \\ x_{+}^{\alpha-1} E_{1, \alpha}\left(x_{+}\right) & \text {if } \alpha>1, \\ \frac{x_{+}^{\alpha-1}}{\Gamma(\alpha)}+\cdots+\frac{x_{+}^{\alpha+m-2}}{\Gamma(\alpha+m-1)}+x_{+}^{\alpha+m-1} E_{1, \alpha+m}\left(x_{+}\right) & \text {if } \alpha<1 \text { but } \alpha \neq 0,-1,-2, \cdots\end{cases}
$$


where $m$ is a positive integer satisfying $1<\alpha+m<2$ and

$$
E_{\alpha, \beta}(z)=\sum_{k=0}^{\infty} \frac{z^{k}}{\Gamma(\alpha k+\beta)}
$$

is a two-parameter function of the Mittag-Leffler type.

It follows from the Mittag-Leffler function that

$$
\begin{aligned}
& E_{1,1}(z)=\sum_{k=0}^{\infty} \frac{z^{k}}{\Gamma(k+1)}=\sum_{k=0}^{\infty} \frac{z^{k}}{k !}=e^{z} \\
& E_{1,2}(z)=\sum_{k=0}^{\infty} \frac{z^{k}}{\Gamma(k+2)}=\frac{1}{z} \sum_{k=0}^{\infty} \frac{z^{k+1}}{(k+1) !}=\frac{e^{z}-1}{z} \\
& E_{2,1}\left(z^{2}\right)=\sum_{k=0}^{\infty} \frac{z^{2 k}}{\Gamma(2 k+1)}=\sum_{k=0}^{\infty} \frac{z^{2 k}}{(2 k) !}=\cosh (z)
\end{aligned}
$$

Proof. By Theorem 3.1

$$
f(x)=g(x) * \Phi_{\alpha-1} .
$$

We first assume that $\alpha=1,0,-1,-2, \cdots$, then

$$
f(x)=g(x) * \Phi_{\alpha-1}=g(x) * \delta^{(1-\alpha)}(x)=g^{(1-\alpha)}(x)
$$

in the distributional sense by equation (3).

Consider,

$$
\begin{aligned}
\left(\frac{d}{d x} g(x), \phi(x)\right) & =-\left(g(x), \phi^{\prime}(x)\right)=-\int_{0}^{\infty} e^{x} \phi^{\prime}(x) d x \\
& =-\int_{0}^{\infty} e^{x} d \phi(x)=\phi(0)+\int_{0}^{\infty} g(x) \phi(x) d x
\end{aligned}
$$

which claims

$$
\frac{d}{d x} g(x)=\delta(x)+g(x)
$$

Thus,

$$
\frac{d^{m}}{d x^{m}} g(x)=\delta^{(m-1)}(x)+\cdots+\delta(x)+g(x)
$$

for $m \in Z^{+}$. This implies

$$
g^{(1-\alpha)}(x)=\delta^{(-\alpha)}(x)+\cdots+\delta(x)+g(x)
$$

for $\alpha=0,-1,-2, \cdots$

Let $\lambda=\alpha-1>0$. Obviously,

$$
\begin{aligned}
f(x) & =g(x) * \frac{x_{+}^{\lambda-1}}{\Gamma(\lambda)}=\frac{1}{\Gamma(\lambda)} \int_{0}^{x} e^{\zeta}(x-\zeta)^{\lambda-1} d \zeta \\
& =\frac{1}{\Gamma(\lambda)} \sum_{k=0}^{\infty} \frac{1}{k !} \int_{0}^{x} \zeta^{k}(x-\zeta)^{\lambda-1} d \zeta=\sum_{k=0}^{\infty} \frac{x_{+}^{k+\lambda}}{\Gamma(\lambda+k+1)}
\end{aligned}
$$

using the following identity

$$
\int_{0}^{x} \zeta^{k}(x-\zeta)^{\lambda-1} d \zeta=\frac{\Gamma(k+1) \Gamma(\lambda)}{\Gamma(\lambda+k+1)} x_{+}^{\lambda+k}
$$

This infers that

$$
f(x)=\sum_{k=0}^{\infty} \frac{x_{+}^{k+\lambda}}{\Gamma(\lambda+k+1)}=x_{+}^{\lambda} \sum_{k=0}^{\infty} \frac{x_{+}^{k}}{\Gamma(\lambda+k+1)}=x_{+}^{\lambda} E_{1, \lambda+1}\left(x_{+}\right)=x_{+}^{\alpha-1} E_{1, \alpha}\left(x_{+}\right)
$$


Finally, we assume that $\lambda=\alpha-1<0$ and $\alpha \neq 0,-1,-2, \cdots$,

$$
\begin{aligned}
f(x) & =g(x) * \Phi_{\alpha-1}=\frac{d^{1-\alpha}}{d x^{1-\alpha}} g(x)=g(x) * \frac{x_{+}^{\alpha-2}}{\Gamma(\alpha-1)} \\
& =g(x) * \frac{d^{m}}{d x^{m}} \frac{x_{+}^{\alpha+m-2}}{\Gamma(\alpha+m-1)}=\frac{d^{m}}{d x^{m}} g(x) * \frac{x_{+}^{\alpha+m-2}}{\Gamma(\alpha+m-1)}
\end{aligned}
$$

where $1<\alpha+m<2$. Therefore, we come to

$$
\begin{aligned}
f(x) & =\left(\delta^{(m-1)}(x)+\cdots+\delta(x)\right) * \frac{x_{+}^{\alpha+m-2}}{\Gamma(\alpha+m-1)}+g(x) * \frac{x_{+}^{\alpha+m-2}}{\Gamma(\alpha+m-1)} \\
& =\frac{x_{+}^{\alpha-1}}{\Gamma(\alpha)}+\cdots+\frac{x_{+}^{\alpha+m-2}}{\Gamma(\alpha+m-1)}+g(x) * \frac{x_{+}^{\alpha+m-2}}{\Gamma(\alpha+m-1)} .
\end{aligned}
$$

Evidently,

$$
\begin{aligned}
g(x) * \frac{x_{+}^{\alpha+m-2}}{\Gamma(\alpha+m-1)} & =\frac{1}{\Gamma(\alpha+m-1)} \int_{0}^{x} e^{t}(x-t)^{\alpha+m-2} d t \\
& =\frac{1}{\Gamma(\alpha+m-1)} \sum_{k=0}^{\infty} \frac{1}{k !} \int_{0}^{x} t^{k}(x-t)^{\alpha+m-2} d t \\
& =\sum_{k=0}^{\infty} \frac{x_{+}^{\alpha+m+k-1}}{\Gamma(\alpha+m+k)}=x_{+}^{\alpha+m-1} E_{1, \alpha+m}\left(x_{+}\right) .
\end{aligned}
$$

Therefore,

$$
f(x)=\frac{x_{+}^{\alpha-1}}{\Gamma(\alpha)}+\cdots+\frac{x_{+}^{\alpha+m-2}}{\Gamma(\alpha+m-1)}+x_{+}^{\alpha+m-1} E_{1, \alpha+m}\left(x_{+}\right)=I_{1}+I_{2}
$$

where

is a distribution and

$$
I_{1}=\frac{x_{+}^{\alpha-1}}{\Gamma(\alpha)}+\cdots+\frac{x_{+}^{\alpha+m-2}}{\Gamma(\alpha+m-1)}
$$

$$
I_{2}=x_{+}^{\alpha+m-1} E_{1, \alpha+m}\left(x_{+}\right)
$$

is an ordinary function. This completes the proof of theorem.

In particular, we get for $\alpha=1 / 2($ thus $m=1)$

$$
f(x)=\frac{x_{+}^{-\frac{1}{2}}}{\sqrt{\pi}}+x_{+}^{\frac{1}{2}} E_{1,3 / 2}\left(x_{+}\right) .
$$

Remark 1. Solutions of many applied problems lead to integral equations, which at the first sight have nothing in common with the Abel's integral equation, and due to this impression additional efforts are undertaken for the development of analytical or numerical procedure for solving these equations. However, their transformations to the form of the Abel's integral equations may often be convenient for rapidly obtaining the solutions. Podlubny provided several typical examples of equations in the classical sense, which can be reduced to the Abel's integral equation in [20] and many types of such equations along with solution formulas can be found in [21] and [22].

Theorem 3.3 The following integral equation with non-moving integration limits

$$
\int_{0}^{\infty} \frac{\phi\left(\sqrt{s^{2}+y^{2}}\right)}{s^{2}+y^{2}} d s=\frac{f(y)}{y}
$$

has the solution

$$
\phi\left(\frac{1}{\sqrt{t}}\right)=\frac{2 \sqrt{t}}{\sqrt{\pi}}\left(\frac{d}{d t} f\left(\frac{1}{\sqrt{t}}\right) * \frac{t_{+}^{-\frac{1}{2}}}{\Gamma\left(\frac{1}{2}\right)}\right)
$$


where $f(y)$ is given in $\mathcal{D}^{\prime}\left(R^{+}\right)$.

Proof. Denoting

$$
\frac{\phi(r)}{r^{2}}=F\left(r^{2}\right)
$$

we can rewrite equation (9) as

$$
\int_{0}^{\infty} F\left(s^{2}+y^{2}\right) d s=\frac{f(y)}{y} .
$$

Substitution of variables $x=y^{2}, \zeta=s^{2}$ gives

$$
\int_{0}^{\infty} \zeta^{-1 / 2} F(x+\zeta) d \zeta=\frac{2 f(\sqrt{x})}{\sqrt{x}} .
$$

Then the further substitution $\tau=1 /(x+\zeta)$ leads to

$$
\int_{0}^{1 / x}\left(\frac{1}{\tau}-x\right)^{-1 / 2} F\left(\frac{1}{\tau}\right) \frac{1}{\tau^{2}} d \tau=\frac{2 f(\sqrt{x})}{\sqrt{x}},
$$

making

$$
t=\frac{1}{x}, \quad \psi(\tau)=\tau^{-3 / 2} F\left(\frac{1}{\tau}\right)
$$

we come to

$$
\frac{1}{\Gamma\left(\frac{1}{2}\right)} \int_{0}^{t}(t-\tau)^{-1 / 2} \psi(\tau) d \tau=\frac{1}{\sqrt{\pi}} \int_{0}^{t} \frac{\psi(\tau)}{(t-\tau)^{1 / 2}} d \tau=\frac{2}{\sqrt{\pi}} f\left(\frac{1}{\sqrt{t}}\right)
$$

which is the Abel's integral equation with $\alpha=1 / 2$. Hence

$$
\psi(t)=\frac{2}{\sqrt{\pi}} \frac{d^{\frac{1}{2}}}{d t^{\frac{1}{2}}} f\left(\frac{1}{\sqrt{t}}\right),
$$

and performing backward substitution we obtain the solution of equation (9) in terms of fractional derivative of one half

$$
\phi\left(\frac{1}{\sqrt{t}}\right)=\frac{2 \sqrt{t}}{\sqrt{\pi}} \frac{d^{\frac{1}{2}}}{d t^{\frac{1}{2}}} f\left(\frac{1}{\sqrt{t}}\right)=\frac{2 \sqrt{t}}{\sqrt{\pi}}\left(\frac{d}{d t} f\left(\frac{1}{\sqrt{t}}\right) * \frac{t_{+}^{-\frac{1}{2}}}{\Gamma\left(\frac{1}{2}\right)}\right)
$$

where all the derivatives above are the fractional derivatives in the distributional sense. This completes the proof of theorem.

Remark 2. We would like to add that $\frac{d^{\frac{1}{2}}}{d t^{\frac{1}{2}}} f\left(\frac{1}{\sqrt{t}}\right)$ may exist distributionally but not classically, since the fractional derivatives of distributions are more general. Here is a simple example to illustrate this. Assume that $f(t)=t_{+}^{\frac{5}{2}}$. Then $\frac{d^{\frac{1}{2}}}{d t^{\frac{1}{2}}} f\left(\frac{1}{\sqrt{t}}\right)$ exists in the distributional sense but not classically.

Theorem 3.4 The following integral equation with a moving integration limit

$$
\int_{0}^{y} \frac{1}{\left(y^{2}-x^{2}\right)^{\beta}} \phi(x) d x=f(y)
$$

has the solution

$$
\phi(\sqrt{t})=\frac{2 \sqrt{t}}{\Gamma(1-\beta)}\left(f(\sqrt{t}) * \frac{t_{+}^{\beta-2}}{\Gamma(\beta-1)}\right)
$$

where $f(y)$ is given in $\mathcal{D}^{\prime}\left(R^{+}\right)$.

Proof. Making the substitutions

$$
\tau=x^{2}, \quad t=y^{2},
$$


and writing

$$
\psi(\tau)=\frac{\phi(\sqrt{\tau})}{\sqrt{\tau}},
$$

we come to the Abel's integral equation:

$$
\int_{0}^{t} \frac{1}{(t-\tau)^{\beta}} \psi(\tau) d \tau=2 f(\sqrt{t})
$$

with the solution

$$
\psi(t)=\frac{2}{\Gamma(1-\beta)} \frac{d^{1-\beta}}{d t^{1-\beta}} f(\sqrt{t}),
$$

and the solution of equation is given by the formula

$$
\phi(\sqrt{t})=\frac{2 \sqrt{t}}{\Gamma(1-\beta)} \frac{d^{1-\beta}}{d t^{1-\beta}} f(\sqrt{t})=\frac{2 \sqrt{t}}{\Gamma(1-\beta)}\left(f(\sqrt{t}) * \frac{t_{+}^{\beta-2}}{\Gamma(\beta-1)}\right)
$$

where $\beta$ is arbitrary and the derivative is in distributional sense. This completes the proof of theorem.

Remark 3. This integral equation appears in numerous applied problems and was investigated in [20] in the classical sense.

Theorem 3.5 The following Abel's integral equation

$$
(1-x)^{-\beta} \delta^{(k)}(x)=\frac{1}{\Gamma(1-\alpha)} \int_{0}^{x} \frac{f(\zeta)}{(x-\zeta)^{\alpha}} d \zeta, \quad \alpha \in R
$$

has the solution

$$
f(x)=\sum_{r=0}^{k}(-1)^{r}\left(\begin{array}{l}
k \\
r
\end{array}\right) \frac{\Gamma(\beta+r)}{\Gamma(\beta)} \Phi_{\alpha-1-k+r}
$$

Proof. It follows that for $k=1,2, \cdots$

$$
(1-x)^{-\beta} \delta^{(k)}(x)=\sum_{r=0}^{k}(-1)^{r}\left(\begin{array}{l}
k \\
r
\end{array}\right) \frac{\Gamma(\beta+r)}{\Gamma(\beta)} \delta^{(k-r)}(x)
$$

based on the identity

$$
\phi(x) \delta^{(k)}(x)=\sum_{r=0}^{k}(-1)^{r}\left(\begin{array}{l}
k \\
r
\end{array}\right) \phi^{(r)}(0) \delta^{(k-r)}(x)
$$

if $\phi(x)$ is an infinitely differentiable function at $x=0$. Therefore,

$$
f(x)=(1-x)^{-\beta} \delta^{(k)}(x) * \Phi_{\alpha-1}=\sum_{r=0}^{k}(-1)^{r}\left(\begin{array}{l}
k \\
r
\end{array}\right) \frac{\Gamma(\beta+r)}{\Gamma(\beta)} \Phi_{\alpha-1-k+r} .
$$

This completes the proof of theorem.

Note that if $g$ is the distribution that has support at a single point $x_{0}$, then $g$ is in fact a finite linear combination of the $\delta$ function and its derivatives at $x_{0}$. That is, there exist a nonnegative integer $k$ and constants $c_{i}$ for $i=0,1,2, \cdots, k$ such that

$$
g(x)=\sum_{i=0}^{k} c_{i} \delta^{(i)}\left(x-x_{0}\right)
$$

Therefore, the Abel's integral equation

$$
g(x)=\frac{1}{\Gamma(1-\alpha)} \int_{0}^{x} \frac{f(\zeta)}{(x-\zeta)^{\alpha}} d \zeta, \quad \alpha \in R
$$


has the solution

$$
f(x)=g(x) * \Phi_{\alpha-1}=\sum_{i=0}^{k} c_{i} \Phi_{\alpha-1-i}\left(x-x_{0}\right) .
$$

Clearly, the distribution $(1-x)^{-\beta} \delta^{(k)}(x)$ in Theorem 3.5 is a finite linear combination of the $\delta$ function and its derivatives at the origin.

Theorem 3.6 Let $\beta$ be any real number except all negative integers. Then the Abel's integral equation

$$
x_{+}^{\beta}=\frac{1}{\Gamma(1-\alpha)} \int_{0}^{x} \frac{f(\zeta)}{(x-\zeta)^{\alpha}} d \zeta, \quad \alpha \in R
$$

has the solution

$$
f(x)=\Gamma(\beta+1) \Phi_{\alpha+\beta}(x) .
$$

Proof. It follows from the following identity

$$
x_{+}^{\beta}=\Gamma(\beta+1) \Phi_{\beta+1}(x) .
$$

This completes the proof of theorem.

We would like to point out that the distribution $x_{+}^{\beta}$ is undefined for a negative integer $\beta$.

Remark 4. The hyper-geometric function, written $F(\alpha, \beta, \gamma ; x)$, is defined for $\operatorname{Re} \gamma>\operatorname{Re} \beta>0$ for $|x|<1$ by the integral

$$
F(\alpha, \beta, \gamma ; x)=\frac{\Gamma(\gamma)}{\Gamma(\beta) \Gamma(\gamma-\beta)} \int_{0}^{1} t^{\beta-1}(1-t)^{\gamma-\beta-1}(1-t x)^{-\alpha} d t .
$$

For the remaining values of $\beta, \gamma(\gamma \neq 0,-1,-2, \cdots)$ and $|x|<1$ it is defined by analytic continuation. It was proved in [13] that

$$
F(\alpha,-k, \gamma ; x)=\sum_{r=0}^{k}(-1)^{r}\left(\begin{array}{l}
k \\
r
\end{array}\right) \frac{\Gamma(\alpha+r)}{\Gamma(\alpha)} \frac{\Gamma(\gamma)}{\Gamma(\gamma+r)} x_{+}^{r} .
$$

Hence, we can easily find the solution for the following Abel's integral equation by Theorem 3.6

$$
F(\alpha,-k, \gamma ; x)=\frac{1}{\Gamma(1-\alpha)} \int_{0}^{x} \frac{f(\zeta)}{(x-\zeta)^{\alpha}} d \zeta, \quad \alpha \in R .
$$

Theorem 3.7 Let $\alpha$ be any real number and $\alpha \neq 1,2, \cdots$ and let $g(x)$ be given in $\mathcal{D}^{\prime}\left(R^{+}\right)$. Then the Abel's integral equation (variant)

$$
g(x)=\int_{0}^{x} \frac{f(\zeta)}{(x-\zeta)^{\alpha}} d \zeta
$$

has the solution

$$
f(x)=\frac{1}{\Gamma(1-\alpha) \Gamma(\alpha-1)} g(x) * x_{+}^{\alpha-2}=\frac{1}{\Gamma(1-\alpha) \Gamma(\alpha)} g^{\prime}(x) * x_{+}^{\alpha-1}
$$

where $g^{\prime}(x)$ is in the distributional sense. In particular, we have the classical solution of the Abel's integral equation for $0<\alpha<1$,

$$
f(x)=\frac{\sin \alpha \pi}{\pi} \int_{0}^{x} \frac{g^{\prime}(\zeta)}{(x-\zeta)^{1-\alpha}} d \zeta
$$

where $g^{\prime}(x)$ (the derivative is in the classical sense) is a continuous function on $[0, \infty)$.

Proof. Evidently, Abel's integral equation

$$
g(x)=\int_{0}^{x} \frac{f(\zeta)}{(x-\zeta)^{\alpha}} d \zeta
$$


turns to be for $\alpha \neq 1,2, \cdots$

$$
\frac{1}{\Gamma(1-\alpha)} g(x)=f(x) * \Phi_{1-\alpha}
$$

which infers by equation (6)

$$
\begin{aligned}
f(x) & =\frac{1}{\Gamma(1-\alpha)} g(x) * \Phi_{\alpha-1}=\frac{1}{\Gamma(1-\alpha) \Gamma(\alpha-1)} g(x) * x_{+}^{\alpha-2} \\
& =\frac{1}{\Gamma(1-\alpha)} g(x) * \frac{d}{d x} \frac{x_{+}^{\alpha-1}}{\Gamma(\alpha)}=\frac{1}{\Gamma(1-\alpha) \Gamma(\alpha)} g^{\prime}(x) * x_{+}^{\alpha-1} .
\end{aligned}
$$

Using

for $0<\alpha<1$, we come to

$$
\Gamma(1-\alpha) \Gamma(\alpha)=\frac{\pi}{\sin \alpha \pi}
$$

$$
f(x)=\frac{\sin \alpha \pi}{\pi} \int_{0}^{x} \frac{g^{\prime}(\zeta)}{(x-\zeta)^{1-\alpha}} d \zeta
$$

if $g^{\prime}(x)$ is a continuous function in the classical sense. This completes the proof of theorem.

Clearly, Abel's integral equation in distribution is an extension of ones in the classical sense. Here are examples to illustrate this.

Example 3.1 Let us consider the Abel's integral equation

$$
g(x)=\int_{0}^{x} \frac{f(\zeta)}{(x-\zeta)^{\alpha}} d \zeta, \quad 0<\alpha<1
$$

where $g(x) \in \mathcal{D}^{\prime}\left(R^{+}\right)$is given by

$$
g(x)=\left\{\begin{array}{l}
1 \text { if } x \text { is irrational and positive } \\
0 \text { otherwise }
\end{array}\right.
$$

Then the ordinary derivative of $g(x)$ does not exist, and therefore this equation has no solution in the classical sense. However, the distributional derivative of $g(x)$ does exist and $g^{\prime}(x)=\delta(x)$ based on the following

$$
\left(g^{\prime}(x), \phi(x)\right)=-\left(g(x), \phi^{\prime}(x)\right)=-\int_{0}^{\infty} \phi^{\prime}(x) d x=\phi(0)=(\delta(x), \phi(x))
$$

as the measure of rational numbers is zero. Hence, the distributional solution is

$$
f(x)=\frac{\sin \alpha \pi}{\pi} \delta(x) * x_{+}^{\alpha-1}=\frac{\sin \alpha \pi}{\pi} x_{+}^{\alpha-1} .
$$

Example 3.2 Let us consider the Abel's integral equation

$$
g(x)=\int_{0}^{x} \frac{f(\zeta)}{(x-\zeta)^{\alpha}} d \zeta, \quad 0<\alpha<1
$$

where $g(x) \in \mathcal{D}^{\prime}\left(R^{+}\right)$is given by

$$
g(x)=\left\{\begin{array}{l}
x \text { if } 0<x<1, \\
0 \text { otherwise }
\end{array}\right.
$$

Then the ordinary derivative of $g(x)$ does not exist, and therefore this equation has no solution in the classical sense. However, we are able to find the distributional derivative of $g(x)$. Indeed,

$$
\begin{aligned}
\left(g^{\prime}(x), \phi(x)\right) & =-\left(g(x), \phi^{\prime}(x)\right)=-\int_{0}^{1} x \phi^{\prime}(x) d x \\
& =-\phi(1)+\int_{0}^{1} \phi(x) d x \\
& =-\phi(1)+\int_{-\infty}^{\infty}(\theta(x)-\theta(x-1)) \phi(x) d x \\
& =(-\delta(x-1), \phi(x))+\int_{-\infty}^{\infty}(\theta(x)-\theta(x-1)) \phi(x) d x
\end{aligned}
$$


which implies that

$$
g^{\prime}(x)=-\delta(x-1)+\theta(x)-\theta(x-1)
$$

Therefore,

$$
\begin{aligned}
f(x) & =\frac{\sin \alpha \pi}{\pi}(-\delta(x-1)+\theta(x)-\theta(x-1)) * x_{+}^{\alpha-1} \\
& =-\frac{\sin \alpha \pi}{\pi}(x-1)_{+}^{\alpha-1}+\frac{1}{\Gamma(1-\alpha) \Gamma(\alpha+1)}\left(x_{+}^{\alpha}-(x-1)_{+}^{\alpha}\right) \\
& =-\frac{\sin \alpha \pi}{\pi}(x-1)_{+}^{\alpha-1}+\frac{\sin \alpha \pi}{\alpha \pi}\left(x_{+}^{\alpha}-(x-1)_{+}^{\alpha}\right) .
\end{aligned}
$$

Theorem 3.8 Let $\phi(x)$ be an infinitely differentiable function on $[0, \infty)$ satisfying

$$
\phi(x)=\sum_{k=0}^{\infty} \frac{\phi^{(k)}(0)}{k !} x^{k} .
$$

Then the following Abel's integral equation

$$
\theta(x) \phi(x)=\frac{1}{\Gamma(1-\alpha)} \int_{0}^{x} \frac{f(\zeta)}{(x-\zeta)^{\alpha}} d \zeta, \quad \alpha \in R
$$

has the solution

$$
f(x)= \begin{cases}\theta(x) \phi(x) & \text { if } \alpha=1, \\ \phi(0) \delta^{(-\alpha)}(x)+\phi^{\prime}(0) \delta^{(-\alpha+1)}(x)+\cdots+\phi^{(-\alpha)}(0) \delta(x)+\theta(x) \phi^{(1-\alpha)}(x) & \text { if } \alpha=0,-1,-2, \cdots, \\ \sum_{k=0}^{\infty} \frac{\phi^{(k)}(0) x_{+}^{k+\alpha-1}}{\Gamma(\alpha+k)} & \text { if } \alpha>1, \\ \sum_{k=-m}^{\infty} \frac{\phi^{(m+k)}(0) x_{+}^{k+\alpha+m-1}}{\Gamma(\alpha+m+k)} & \text { if } \alpha<1 \text { but } \alpha \neq 0,-1,-2, \cdots\end{cases}
$$

where $1<\alpha+m<2$ and $m \in Z^{+}$.

Proof. We note that for $m \in Z^{+}$,

$$
\frac{d^{m}}{d x^{m}}(\theta(x) \phi(x))=\phi(0) \delta^{(m-1)}(x)+\cdots+\phi^{(m-1)}(0) \delta(x)+\theta(x) \phi^{(m)}(x) .
$$

Indeed, we have for $m=1$ that

$$
\begin{aligned}
& \left(\frac{d}{d x}(\theta(x) \phi(x)), \psi(x)\right)=-\int_{0}^{\infty} \phi(x) \psi^{\prime}(x) d x=-\left.\phi(x) \psi(x)\right|_{0} ^{\infty}+\int_{0}^{\infty} \phi^{\prime}(x) \psi(x) d x \\
& =\phi(0)(\delta(x), \psi(x))+\int_{0}^{\infty} \phi^{\prime}(x) \psi(x) d x=\phi(0)(\delta(x), \psi(x))+\left(\theta(x) \phi^{\prime}(x), \psi(x)\right)
\end{aligned}
$$

using integration by parts. This indicates

$$
\frac{d}{d x}(\theta(x) \phi(x))=\phi(0) \delta(x)+\theta(x) \phi^{\prime}(x) .
$$

The result follows inductively and the rest is similar to Theorem 3.2. This completes the proof of theorem.

Example 3.3 The following Abel's integral equation

$$
\theta(x) \frac{x}{x^{2}+b^{2}}=\int_{0}^{x} \frac{f(\zeta)}{(x-\zeta)^{\frac{1}{2}}} d \zeta, \quad b>0 \quad \text { and } \quad x<b
$$

has the solution

$$
f(x)=\sum_{k=0}^{\infty} \frac{(-1)^{k} 4^{2 k+1}[(2 k+1) !]^{2} x_{+}^{2 k+1 / 2}}{b^{2 k+2}(4 k+2) !} .
$$


Proof. Let

$$
\phi(x)=\frac{x}{x^{2}+b^{2}} .
$$

Then $\phi(0)=0$. By Theorem 3.8, we come to

$$
f(x)=\sqrt{\pi} \sum_{k=-1}^{\infty} \frac{\phi^{(k+1)}(0) x_{+}^{k+1 / 2}}{\Gamma(1 / 2+1+k)}=\sqrt{\pi} \sum_{k=0}^{\infty} \frac{\phi^{(k+1)}(0) x_{+}^{k+1 / 2}}{\Gamma(1 / 2+1+k)}
$$

since $m=1$ and $\alpha=1 / 2$.

It follows from [23] that

$$
\frac{d^{k+1}}{d x^{k+1}}\left(\frac{x}{x^{2}+b^{2}}\right)=\frac{(-1)^{k+1}(k+1) !}{\left(x^{2}+b^{2}\right)^{k+2}} \sum_{0 \leq 2 i \leq k+2}(-1)^{i}\left(\begin{array}{c}
k+2 \\
2 i
\end{array}\right) b^{2 i} x^{k+2-2 i}
$$

which implies that

$$
\begin{aligned}
& \left.\frac{d^{2 k+1}}{d x^{2 k+1}}\left(\frac{x}{x^{2}+b^{2}}\right)\right|_{x=0}=\frac{(-1)^{k}(2 k+1) !}{b^{2 k+2}}, \text { and } \\
& \left.\frac{d^{2 k}}{d x^{2 k}}\left(\frac{x}{x^{2}+b^{2}}\right)\right|_{x=0}=0 .
\end{aligned}
$$

Using the formula

$$
\Gamma(k+1 / 2)=\frac{\sqrt{\pi}(2 k) !}{2^{2 k} k !},
$$

we have

$$
\begin{aligned}
f(x) & =\sqrt{\pi} \sum_{k=0}^{\infty} \frac{\phi^{(k+1)}(0) x_{+}^{k+1 / 2}}{\Gamma(1 / 2+1+k)} \\
& =\sqrt{\pi} \sum_{k=0}^{\infty} \frac{(-1)^{k}(2 k+1) ! x_{+}^{2 k+1 / 2}}{b^{2 k+2} \Gamma(1 / 2+2 k+1)} \\
& =\sum_{k=0}^{\infty} \frac{(-1)^{k} 4^{2 k+1}[(2 k+1) !]^{2} x_{+}^{2 k+1 / 2}}{b^{2 k+2}(4 k+2) !} .
\end{aligned}
$$

This completes the proof of example.

There is a simpler approach to finding $\phi^{(k+1)}(0)$. Indeed,

$$
\begin{aligned}
\phi(x) & =\frac{x}{x^{2}+b^{2}}=\frac{x}{b^{2}\left(1+\left(\frac{x}{b}\right)^{2}\right)} \\
& =\frac{x}{b^{2}} \sum_{k=0}^{\infty}(-1)^{k}\left(\frac{x}{b}\right)^{2 k}=\sum_{k=0}^{\infty}(-1)^{k} \frac{x^{2 k+1}}{b^{2 k+2}},
\end{aligned}
$$

which also infers that

$$
\phi^{(2 k+1)}(0)=\frac{(-1)^{k}(2 k+1) !}{b^{2 k+2}} \quad \text { and } \quad \phi^{(2 k)}(0)=0 .
$$

Similarly, the following Abel's integral equation which is undefined in the classical sense

$$
\theta(x) \frac{x}{x^{2}+b^{2}}=\int_{0}^{x} \frac{f(\zeta)}{(x-\zeta)^{\frac{3}{2}}} d \zeta, \quad b>0 \quad \text { and } \quad x<b
$$


has the solution

$$
\begin{aligned}
f(x) & =\Gamma(-1 / 2) \sum_{k=0}^{\infty} \frac{\phi^{(k)}(0) x_{+}^{k+1 / 2}}{\Gamma(k+3 / 2)}=-2 \sqrt{\pi} \sum_{k=0}^{\infty} \frac{(-1)^{k}(2 k+1) ! x_{+}^{2 k+3 / 2}}{b^{2 k+2} \Gamma(2 k+1+3 / 2)} \\
& =\sum_{k=0}^{\infty} \frac{(-1)^{k+1} 2^{4 k+5}(2 k+1) !(2 k+2) ! x_{+}^{2 k+3 / 2}}{b^{2 k+2}(4 k+4) !}
\end{aligned}
$$

by using

$$
\begin{aligned}
& \Gamma(-1 / 2)=-2 \sqrt{\pi}, \quad \text { and } \\
& \Gamma(2 k+1+3 / 2)=\frac{\sqrt{\pi}(4 k+4) !}{2^{4 k+4}(2 k+2) !} .
\end{aligned}
$$

Furthermore, we can solve the following Abel's integral equations

$$
\begin{array}{ll}
\theta(x) \arcsin x=\int_{0}^{x} \frac{f(\zeta)}{(x-\zeta)^{\frac{1}{2}}} d \zeta, & 0<x<1 \quad \text { or } \\
\theta(x) \arcsin x=\int_{0}^{x} \frac{f(\zeta)}{(x-\zeta)^{\frac{3}{2}}} d \zeta, & 0<x<1
\end{array}
$$

by using the Taylor's series

$$
\arcsin x=\sum_{n=0}^{\infty} \frac{(2 n) !}{4^{n}(n !)^{2}(2 n+1)} x^{2 n+1}, \quad \text { for } \quad 0<x<1 .
$$

based on the above example.

Theorem 3.9 Let $\phi(x) \in C^{m}[0, \infty)$. Then the following Abel's integral equation

$$
\theta(x) \phi(x)=\frac{1}{\Gamma(1-\alpha)} \int_{0}^{x} \frac{f(\zeta)}{(x-\zeta)^{\alpha}} d \zeta, \quad \alpha \in R
$$

has the solution

$$
f(x)= \begin{cases}\frac{d^{1-\alpha}}{d x^{1-\alpha}}(\theta(x) \phi(x)) & \text { if } \alpha=1,0,-1,-2, \cdots, \\ \frac{1}{\Gamma(\alpha-1)} \int_{0}^{x} \phi(\zeta)(x-\zeta)^{\alpha-2} d \zeta & \text { if } \alpha>1, \\ F(x) & \text { if } \alpha<1 \text { but } \alpha \neq 0,-1,-2 . \cdots\end{cases}
$$

where $m$ is a positive integer satisfying $1<\alpha+m<2$, and

$$
\begin{aligned}
F(x)= & \phi(0) \frac{x_{+}^{\alpha-1}}{\Gamma(\alpha)}+\cdots+\phi^{(m-1)}(0) \frac{x_{+}^{m+\alpha-2}}{\Gamma(m+\alpha-1)} \\
& +\frac{1}{\Gamma(m+\alpha-1)} \int_{0}^{x} \phi^{(m)}(t)(x-t)^{m+\alpha-2} d t .
\end{aligned}
$$

Proof. For $\alpha=1,0,-1, \cdots$, we have

$$
\theta(x) \phi(x)=\frac{1}{\Gamma(1-\alpha)} \int_{0}^{x} \frac{f(\zeta)}{(x-\zeta)^{\alpha}} d \zeta=f * \Phi_{1-\alpha}
$$

which implies that

$$
f=(\theta(x) \phi(x)) * \Phi_{\alpha-1}=(\theta(x) \phi(x)) * \delta^{(1-\alpha)}(x)=\frac{d^{1-\alpha}}{d x^{1-\alpha}}(\theta(x) \phi(x))
$$


where $\frac{d^{1-\alpha}}{d x^{1-\alpha}}(\theta(x) \phi(x))$ is in the distributional sense. That is,

$$
\left(\frac{d^{1-\alpha}}{d x^{1-\alpha}}(\theta(x) \phi(x)), \psi(x)\right)=(-1)^{1-\alpha}\left(\theta(x) \phi(x), \psi^{(1-\alpha)}(x)\right)
$$

where $\psi(x)$ is in $\mathcal{D}(R)$.

For example, if we let $\phi(x)=\sqrt{x} \in C[0, \infty)$ then

$$
\frac{d^{1-\alpha}}{d x^{1-\alpha}}(\theta(x) \sqrt{x})=\frac{d^{1-\alpha}}{d x^{1-\alpha}} x_{+}^{\frac{1}{2}}=\Gamma(1 / 2+1) \frac{d^{1-\alpha}}{d x^{1-\alpha}} \frac{x_{+}^{\frac{1}{2}}}{\Gamma(1 / 2+1)}=\frac{\sqrt{\pi}}{2 \Gamma(1 / 2+\alpha)} x_{+}^{-1 / 2+\alpha} .
$$

Secondly, we assume that $\alpha>1$ then

$$
f(x)=(\theta(x) \phi(x)) * \Phi_{\alpha-1}=\frac{1}{\Gamma(\alpha-1)} \int_{0}^{x} \phi(\zeta)(x-\zeta)^{\alpha-2} d \zeta .
$$

Finally, we suppose $\alpha<1$ and $\alpha \neq 0,-1, \cdots$. Let $\rho(x)$ be the infinitely differentiable function given by

$$
\rho(x)= \begin{cases}\mathrm{c} \exp \left(-\frac{1}{1-x^{2}}\right) & \text { if }|x|<1, \\ 0 & \text { if }|x| \geq 1\end{cases}
$$

where $c$ is the constant such that $\int_{-1}^{1} \rho(x) d x=1$. Obviously, the sequence (a particular form of the $\delta$-sequence used in [24])

$$
\delta_{n}(x)=n \rho(n x)
$$

is an infinitely differentiable sequence converging to $\delta(x)$ in $\mathcal{D}^{\prime}(R)$ as $n \rightarrow \infty$.

Let $\phi_{1}(x) \in C^{m}(R)$ such that $\phi_{1}(x)=\phi(x)$ for $x \in[0, \infty)$. Then the convolution given by

$$
\psi_{n}(x)=\phi_{1}(x) * \delta_{n}(x)=\int_{-\infty}^{\infty} \phi_{1}(x-y) \delta_{n}(y) d y
$$

is an infinitely differentiable sequence and uniformly converges to $\phi_{1}(x)$ as $n \rightarrow \infty$ on every compact subset $L \subset R$. Indeed, $\phi_{1}(x)$ is uniformly continuous on $L$ since it is continuous on $L$ and $L$ is compact. Therefore, for all $\epsilon>0$ there exists $\delta>0$, such that

$$
\left|\phi_{1}(x-y)-\phi_{1}(x)\right|<\epsilon
$$

for all $x \in L$ and $|y|<\delta$. Choosing $n>1 / \delta$, we arrive at

$$
\left|\psi_{n}(x)-\phi_{1}(x)\right| \leq \int_{-\infty}^{\infty}\left|\phi_{1}(x-y)-\phi_{1}(x)\right| \delta_{n}(y) d y<\epsilon
$$

holds for all $x \in L$. Similarly, we can prove that $\psi_{n}^{(k)}(x)$ uniformly converges to $\phi_{1}^{(k)}(x)$ on every compact subset of $R$ for $0 \leq k \leq m$.

It follows by Theorem 3.8 that

$$
\frac{d^{m}}{d x^{m}}\left(\theta(x) \psi_{n}(x)\right)=\psi_{n}(0) \delta^{(m-1)}(x)+\cdots+\psi_{n}^{(m-1)}(0) \delta(x)+\theta(x) \psi_{n}^{(m)}(x)
$$

since $\psi_{n}(x)$ is an infinitely differentiable sequence. Clearly,

$$
\lim _{n \rightarrow \infty} \int_{-\infty}^{\infty} \theta(x) \psi_{n}^{(m)}(x) \varphi(x) d x=\int_{0}^{\infty} \phi^{(m)}(x) \varphi(x) d x
$$

for all $\varphi(x) \in \mathcal{D}(R)$ and

$$
\begin{aligned}
& \lim _{n \rightarrow} \psi_{n}(0)=\phi_{1}(0)=\phi(0), \\
& \ldots \\
& \lim _{n \rightarrow} \psi_{n}^{(m-1)}(0)=\phi_{1}^{(m-1)}(0)=\phi^{(m-1)}(0) .
\end{aligned}
$$


Therefore,

$$
\frac{d^{m}}{d x^{m}}(\theta(x) \phi(x))=\phi(0) \delta^{(m-1)}(x)+\cdots+\phi^{(m-1)}(0) \delta(x)+\theta(x) \phi^{(m)}(x) .
$$

for all $\phi(x) \in C^{m}[0, \infty)$, which infers that for $1<\alpha+m<2$

$$
\begin{aligned}
f(x)= & (\theta(x) \phi(x)) * \Phi_{\alpha-1}=(\theta(x) \phi(x)) * \frac{d^{m}}{d x^{m}} \frac{x_{+}^{\alpha+m-2}}{\Gamma(\alpha+m-1)} \\
= & \frac{d^{m}}{d x^{m}}(\theta(x) \phi(x)) * \frac{x_{+}^{\alpha+m-2}}{\Gamma(\alpha+m-1)} \\
= & \left(\phi(0) \delta^{(m-1)}(x)+\cdots+\phi^{(m-1)}(0) \delta(x)+\theta(x) \phi^{(m)}(x)\right) * \frac{x_{+}^{\alpha+m-2}}{\Gamma(\alpha+m-1)} \\
= & \phi(0) \frac{x_{+}^{\alpha-1}}{\Gamma(\alpha)}+\cdots+\phi^{(m-1)}(0) \frac{x_{+}^{\alpha+m-2}}{\Gamma(\alpha+m-1)} \\
& +\frac{1}{\Gamma(m+\alpha-1)} \int_{0}^{x} \phi^{(m)}(t)(x-t)^{m+\alpha-2} d t=F(x) .
\end{aligned}
$$

This completes the proof of theorem.

Example 3.4 Consider the function given by

$$
\phi(x)=\left\{\begin{array}{l}
x \text { if } 0 \leq x \leq 1 \\
1 \text { if } x>1
\end{array}\right.
$$

and $\alpha=0$ in the integral equation

$$
\theta(x) \phi(x)=\frac{1}{\Gamma(1-\alpha)} \int_{0}^{x} \frac{f(\zeta)}{(x-\zeta)^{\alpha}} d \zeta, \quad \alpha \in R
$$

Then by Theorem 3.9 we derive that

$$
f(x)=\frac{d}{d x}(\theta(x) \phi(x)) .
$$

A direct computation shows that

$$
\begin{aligned}
& \left(\frac{d}{d x}(\theta(x) \phi(x)), \psi(x)\right)=-\left(\theta(x) \phi(x), \psi^{\prime}(x)\right)=-\int_{0}^{\infty} \phi(x) \psi^{\prime}(x) d x \\
& =-\int_{0}^{1} x \psi^{\prime}(x) d x-\int_{1}^{\infty} \psi^{\prime}(x) d x=-\psi(1)+\int_{0}^{1} \psi(x) d x+\psi(1) \\
& =\int_{-\infty}^{\infty}(\theta(x)-\theta(x-1)) \psi(x) d x
\end{aligned}
$$

where $\psi(x) \in \mathcal{D}(R)$. This means that

$$
\frac{d}{d x}(\theta(x) \phi(x))=\theta(x)-\theta(x-1) .
$$

Note that the function $\phi(x)$ is not differentiable at $x=1$ in the classical sense and all above derivatives are in the distributional sense.

Clearly, if $\alpha=-1$ then

$$
f(x)=\frac{d^{2}}{d x^{2}}(\theta(x) \phi(x))=\delta(x)-\delta(x-1) .
$$

Furthermore, we let $\phi(x)=\sqrt{x} \in C[0, \infty)$ and $\alpha=3 / 2$ in Theorem 3.9. Then

$$
f(x)=\frac{1}{\Gamma(1 / 2)} \int_{0}^{x} \zeta^{1 / 2}(x-\zeta)^{-1 / 2} d \zeta=\frac{1}{\Gamma(1 / 2)} \frac{\Gamma(3 / 2) \Gamma(1 / 2)}{\Gamma(2)} x_{+}=\frac{\sqrt{\pi}}{2} x_{+} .
$$


At the end, we suppose that $\phi(x)=x e^{x}$ (note that this function is in $C^{\infty}(R)$ ), and $\alpha<1$ with $\alpha \neq 0,-1, \cdots$ in Theorem 3.9. Then we get for $1<\alpha+m<2$,

$$
\begin{aligned}
f(x)= & \phi(0) \frac{x_{+}^{\alpha-1}}{\Gamma(\alpha)}+\cdots+\phi^{(m-1)}(0) \frac{x_{+}^{\alpha+m-2}}{\Gamma(\alpha+m-1)} \\
& +\frac{1}{\Gamma(m+\alpha-1)} \int_{0}^{x} \phi^{(m)}(t)(x-t)^{m+\alpha-2} d t .
\end{aligned}
$$

Using

$$
\phi^{(m)}(x)=(m+x) e^{x},
$$

we come to

$$
\begin{aligned}
f(x)= & \frac{x_{+}^{\alpha}}{\Gamma(\alpha+1)}+\cdots+(m-1) \frac{x_{+}^{\alpha+m-2}}{\Gamma(\alpha+m-1)} \\
& +\frac{1}{\Gamma(m+\alpha-1)} \int_{0}^{x}(m+t) e^{t}(x-t)^{m+\alpha-2} d t .
\end{aligned}
$$

Clearly,

$$
\begin{aligned}
\frac{1}{\Gamma(m+\alpha-1)} \int_{0}^{x}(m+t) e^{t}(x-t)^{m+\alpha-2} d t= & \frac{1}{\Gamma(m+\alpha-1)} \int_{0}^{x} m e^{t}(x-t)^{m+\alpha-2} d t \\
& +\frac{1}{\Gamma(m+\alpha-1)} \int_{0}^{x} t e^{t}(x-t)^{m+\alpha-2} d t \\
= & m \sum_{k=0}^{\infty} \frac{1}{\Gamma(k+m+\alpha)} x_{+}^{k+m+\alpha-1} \\
& +\sum_{k=0}^{\infty} \frac{k+1}{\Gamma(k+m+\alpha+1)} x_{+}^{k+m+\alpha} .
\end{aligned}
$$

Hence,

$$
\begin{aligned}
f(x)= & \frac{x_{+}^{\alpha}}{\Gamma(\alpha+1)}+\cdots+(m-1) \frac{x_{+}^{\alpha+m-2}}{\Gamma(\alpha+m-1)} \\
& +m \sum_{k=0}^{\infty} \frac{1}{\Gamma(k+m+\alpha)} x_{+}^{k+m+\alpha-1} \\
& +\sum_{k=0}^{\infty} \frac{k+1}{\Gamma(k+m+\alpha+1)} x_{+}^{k+m+\alpha} .
\end{aligned}
$$

Acknowledgments. This work is partially supported by NSFC under grant no. 11372170 and BURC.

\section{References}

1. C. J. Cremers and R. C. Birkebak, "Applications of the abel's integral equation to spectrographic data," Appl. Opt., vol. 5, no. 6, pp. 1057-1064, 1966.

2. R. Gorenflo and S. Vessella, Abel Integral Equations: Analysis and Applications. Springer-Verlag, 1991.

3. V. Mirčeski and Z. Tomovski, "Analytical solutions of integral equations for modeling of reversible electrode processes under volumetric conditions," J. Electroanal. Chem., vol. 619-620, pp. 164-168, 2008.

4. N. H. Abel, "Solution de quelques problèmes à i'aide d'intégrales définies," Magaziu for Naturvidenskaberue, Alu-gang I, vol. Bind 2, Christiania, pp. 11-18, 1823.

5. J. D. Tamarkin, "On integrable solutions of abel's integral equation," Annals of Mathematics, vol. 31, pp. 219-229, 1930.

6. D. B. Sumnar, "Abel's integral equation as a convolution of transform," Proceedings of the American Mathematical Society, vol. 7, pp. 82-86, 1956. 
7. G. N. Minerbo and M. E. Levy, "Inversion of abel's integral equation by means of orthogonal polynomials," SIAM Journal on Numerical Analysis, vol. 6, pp. 598-616, 1969.

8. J. R. Hatcher, "A nonlinear boundary problem," Proceedings of the American Mathematical Society, vol. 95, no. 1 , pp. $441-448,1985$.

9. O. P. Singh, V. K. Singh, and R. K. Pandey, "A stable numerical inversion of abel's integral equation using almost bernstein operational matrix," Journal of Quantitative Spectroscopy and Radiative Transfer, vol. 111, pp. 245-252, 2010.

10. M. Li and W. Zhao, "Solving abel's type integral equation with mikusinski's operator of fractional order," Advances in Mathematical Physics, vol. 2013, Article ID 806984, pp. 1-4, 2013.

11. S. Jahanshahi, E. Babolian, D. Torres, and A. Vahidi, "Solving abel integral equations of first kind via fractional calculus," Journal of King Saud University - Science, vol. 27, pp. 161-167, 2015.

12. M. H. Saleh, S. M. Amer, D. S. Mohamed, and A. E. Mahdy, "Identification of common molecular subsequences," International Journal of Computer Applications, vol. 100, pp. 19-23, 2014.

13. I. M. Gel'fand and G. E. Shilov, Generalized Functions. Vol I, Academic Press, New York, 1964.

14. C. Li, "Several results of fractional derivatives in $\mathcal{D}^{\prime}\left(R^{+}\right)$," Fractional Calculus and Applied Analysis, vol. 18, pp. 192-207, 2015.

15. C. P. Li, D. Qian, and Y. Chen, "On riemann-liouville and caputo derivatives," Discrete Dynamics in Nature and Society, vol. 2011, Article ID 562494, pp. 1-15, 2011.

16. C. P. Li and Z. Zhao, "Introduction to fractional integrability and differentiability," The European Physical Journal-Special Topics, vol. 193, pp. 5-26, 2011.

17. C. P. Li and W. Deng, "Remarks on fractional derivatives," Appl. Math. Comput., vol. 187, pp. 777-784, 2007.

18. A. A. Kilbas, H. M. Srivastava, and J. J. Trujillo, Theory and Applications of Fractional Differential Equations. Elsevier, New York, 2006.

19. C. K. Li and C. P. Li, "On defining the distributions $(\delta)^{k}$ and $\left(\delta^{\prime}\right)^{k}$ by fractional derivatives," Applied Mathematics and Computation, vol. 248, pp. 502-513, 2014.

20. I. Podlubny, Fractional Differential Equations. Academic Press, New Yor, 1999.

21. H. M. Srivastava and R. G. Buschman, Theory and Applications of Convolution Integral Equations. Kluwer Academic Publishers, Dordrecht-Boston-London, 1992.

22. X. J. Yang, D. Baleanu, and H. M. Srivastava, Local Fractional Integral Transforms and Their Applications. Academic Press (Elsevier Science Publishers), Amsterdam, Heidelberg, London and New York, 2016.

23. I. S. Gradshteyn and I. M. Ryzhik, Tables of Integrals, Series, and Products. Academic Press, 1980.

24. C. K. Li and C. P. Li, "Remarks on fractional derivatives of distributions," to appear in Tbilisi Mathematical Journal. 